University of Nebraska - Lincoln

DigitalCommons@University of Nebraska - Lincoln

October 1984

\title{
Surface-Wave-Induced Interference Effects in Angle-Resolved Photoemission
}

\author{
Peter A. Dowben \\ University of Nebraska-Lincoln, pdowben@unl.edu \\ D. Heskett \\ University of Pennsylvania-Philadelphia \\ E.W. Plummer \\ University of Pennsylvania-Philadelphia \\ Y. Sakisaka \\ Cornell University
}

T.N. Rhodin

Cornell University

See next page for additional authors

Follow this and additional works at: https://digitalcommons.unl.edu/physicsdowben

Part of the Physics Commons

Dowben, Peter A.; Heskett, D.; Plummer, E.W.; Sakisaka, Y.; Rhodin, T.N.; and Umrigar, Cyrus, "SurfaceWave-Induced Interference Effects in Angle-Resolved Photoemission" (1984). Peter Dowben Publications. 70.

https://digitalcommons.unl.edu/physicsdowben/70

This Article is brought to you for free and open access by the Research Papers in Physics and Astronomy at DigitalCommons@University of Nebraska - Lincoln. It has been accepted for inclusion in Peter Dowben Publications by an authorized administrator of DigitalCommons@University of Nebraska - Lincoln. 


\section{Authors}

Peter A. Dowben, D. Heskett, E.W. Plummer, Y. Sakisaka, T.N. Rhodin, and Cyrus Umrigar 


\title{
Surface-Wave-Induced Interference Effects in Angle-Resolved Photoemission
}

\author{
P. A. Dowben ${ }^{(a)}$ \\ Fritz-Haber-Institut der Max-Planck-Gesellschaft, D-1000 Berlin 33, Federal Republic of Germany \\ and \\ D. Heskett and E. W. Plummer \\ Physics Department, University of Pennsylvania, Philadelphia, Pennsylvania 19104 \\ and \\ Y. Sakisaka ${ }^{(b)}$ and T. N. Rhodin \\ School of Applied and Engineering Physics and The Materials Science Center, \\ Cornell University, Ithaca, New York 14853 \\ and \\ Cyrus Umrigar \\ Laboratory of Atomic and Solid State Physics, Cornell University, Ithaca, New York 14853 \\ (Received 1 March 1984)
}

\begin{abstract}
New features are observed in normal-emission photoelectron spectra from $\mathrm{Ni}(100)$ in a narrow range of photon energies around $25 \mathrm{eV}$. These features are inconsistent with either direct transitions from the bulk or emission from occupied surface states or resonances. We suggest that they are a consequence of interference between the ordinary direct transition emitting an electron in the normal direction and the excitation from the same initial state into a final state that would normally be emitted from the surface at $\bar{\Gamma}$ in the second surface Brillouin zone, but at this energy is trapped in a surface wave.
\end{abstract}

PACS numbers: $79.60 . \mathrm{Cn}$

One of the major accomplishments of angleresolved photoemission using synchrotron radiation has been the ability to measure the dispersion of both the surface and bulk electronic states of a crystalline solid. ${ }^{1-6}$ One of the most extensively studied crystals has been $\mathrm{Ni}(100) .{ }^{1-7}$ Yet we report in this Letter features in the normal emission spectra from this surface which have not been observed previously. These features are readily obscured by trace amounts of impurities which is undoubtedly the reason that they are absent in previous works. ${ }^{1,3-5}$

Figures 1 and 2 illustrate the salient features of the normal emission spectra from $\mathrm{Ni}(100)$ for the discussion included in this Letter. Figure 1, curve $a$, is the spectrum taken at $\hbar \omega=25 \mathrm{eV}$ showing two peaks at $0.5-$ and $1.8-\mathrm{eV}$ binding energy. Curves $b$, $c$, and $d$ of this figure show the results of slight contamination of the surface. The most obvious effect is that the valley in spectrum $a$ (at $\sim 1.0 \mathrm{eV}$ ) gradually fills in as the surface becomes contaminated. Spectrum $d$ resembles previously reported spectra of the allegedly clean $\mathrm{Ni}(100)$ surface. ${ }^{1,5}$ Figure 2 shows the photon energy dependence of the structure shown in Fig. 1. The surface-sensitive valley observed at $\hbar \omega=25 \mathrm{eV}$ has totally disap- peared when $\hbar \omega$ is increased to $30 \mathrm{eV}$ or lowered to $22 \mathrm{eV}$. The dashed curve in Fig. 2 at $25 \mathrm{eV}$ is the spectrum after an equivalent surface contamination as in Fig. 1, curve $d$. The four important properties of these features are the following: (1) The new structure seen at $\hbar \omega=25 \mathrm{eV}$ is very sensitive to surface cleanliness; (2) as the surface is contaminated the valley between the two peaks fills in; (3) this effect is only seen for a narrow range of photon energies and; (4) the peak at $\sim 2.0-\mathrm{eV}$ binding energy disperses as a function of $\hbar \omega$ (see Fig. 2). The peak therefore disperses versus $k_{\perp}$ which shows that it is not a surface state or resonance in the standard sense; nevertheless, it is very surface sensitive. While some contribution to the $0.5-\mathrm{eV}$ bindingenergy feature results from a surface resonance previously reported by Eberhardt and Plummer, ${ }^{1}$ the new structure is not easily explained by the $\mathrm{Ni}(100)$ initial-state surface band structure. The new spectral features most likely result from interference between two different channels of excitation, one of which is severely attenuated by surface contamination.

The experiments reported were performed on a clean $\mathrm{Ni}(100)$ surface prepared by repeated $\mathrm{Ar}^{+}$. 


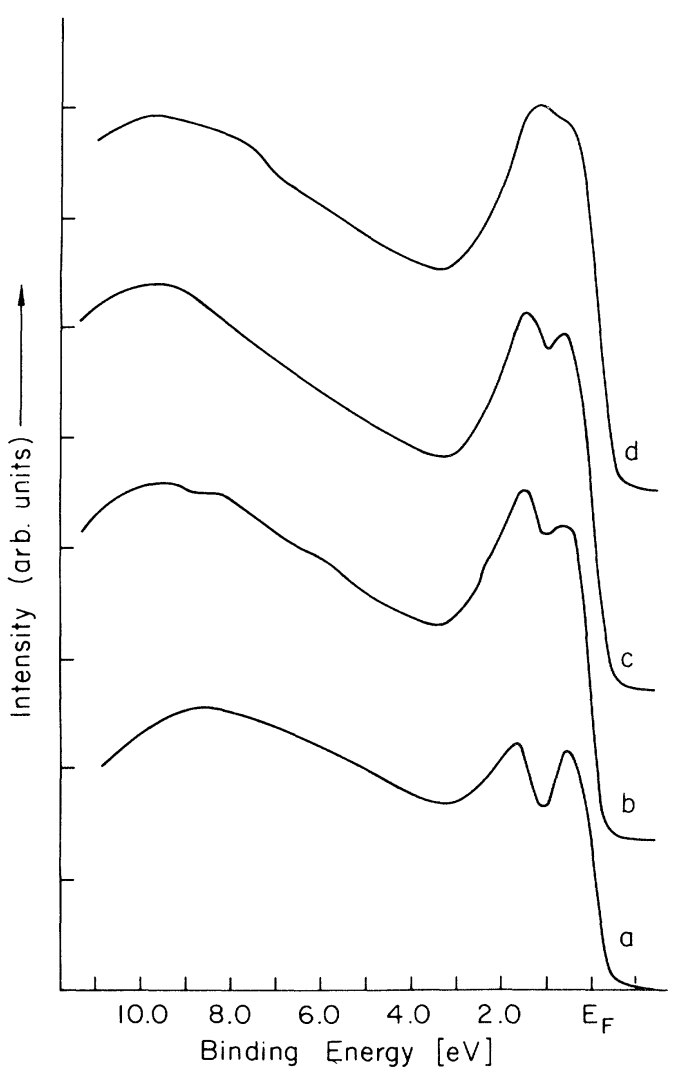

FIG. 1. Angle-resolved photoemission spectra showing the surface-sensitive emission feature for (curve $a$ ) the clean $\mathrm{Ni}(100)$ surface, (curve $b$ ) following 20-min exposure to the ambient atmosphere (mostly $\mathrm{CO}$ ) at pressures less than $2 \times 10^{-10}$ Torr ( $<<2 \%$ of a monolayer), (curve $c$ ) following 40-min exposure to the ambient atmosphere ( $<<4 \%$ of a monolayer), and (curve d) less than 50 -min exposure to the ambient atmosphere ( $<<5 \%$ of a monolayer).

ion bombardment, annealing, and exposure to reactive gases such as NO. The cleanliness of the surface was judged by the behavior of weakly bound adsorbates such as $\mathrm{N}_{2}$ with respect to coverage and heats of adsorption, which was found to be more sensitive to contamination than Auger or photoemission spectroscopies. ${ }^{8}$

The radiation source was the $240-\mathrm{MeV}$ storage ring at the University of Wisconsin, Madison, Synchrotron Radiation Center. The light was dispersed by a $3-\mathrm{m}$ toroidal-grating monochromator. Angleresolved energy distribution curves were taken with an ADES-400 spectrometer (VG Scientific) using a total energy resolution of $0.2-0.3 \mathrm{eV}$ with an angular acceptance of $3^{\circ}$. Throughout the experiments reported here, the photoemitted electrons were collected normal to the $\mathrm{Ni}(100)$ surface.

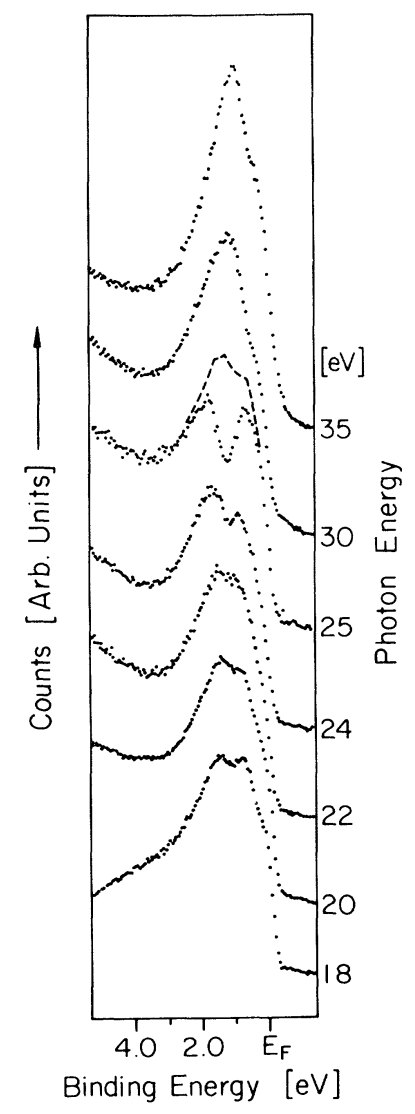

FIG. 2. A set of angle-resolved photoemission spectra showing the photon energy dependence for normal emission from $\mathrm{Ni}(100)$. Light is incident at $45^{\circ}$. The electric vector $\overrightarrow{\mathrm{A}}$ is parallel to the $\bar{\Gamma}-\bar{M}$ line of the surface Brillouin zone. The dashed curve at $25 \mathrm{eV}$ is after slight surface contamination.

The LEED pattern and the surface Brillouin zone of $\mathrm{Ni}(100)$ are shown in Fig. 3(a), with a coordinate system that has the $z$ axis normal to the surface. Normal-emission measurements probe transitions that occur along the $\Delta_{1}$ axis in the bulk band structure, which is shown in Fig. 3(b). This figure shows only those bands which can be coupled in a normal-emission experiment. ${ }^{1,2}$ The only allowed final bands must be the totally symmetric $\Delta_{1}$ bands. ${ }^{1,2}$ The occupied bands shown are those that have been determined experimentally., 5

The dashed curve in the top of Fig. 3(b) is the free-electron band folded back into the first Brillouin zone with a reciprocal lattice vector $G=(2 \pi / a)(0,0,2)$, parallel to the $z$ axis. The dot-dashed bands shown in this figure are freeelectron bands folded back with a reciprocal lattice vector $G=(2 \pi / a)(1,1,1)$ which is not parallel to the normal direction. This latter band is fourfold degenerate and will be split into a $\Delta_{1^{\prime}}, \Delta_{2^{\prime}}$, and two- 


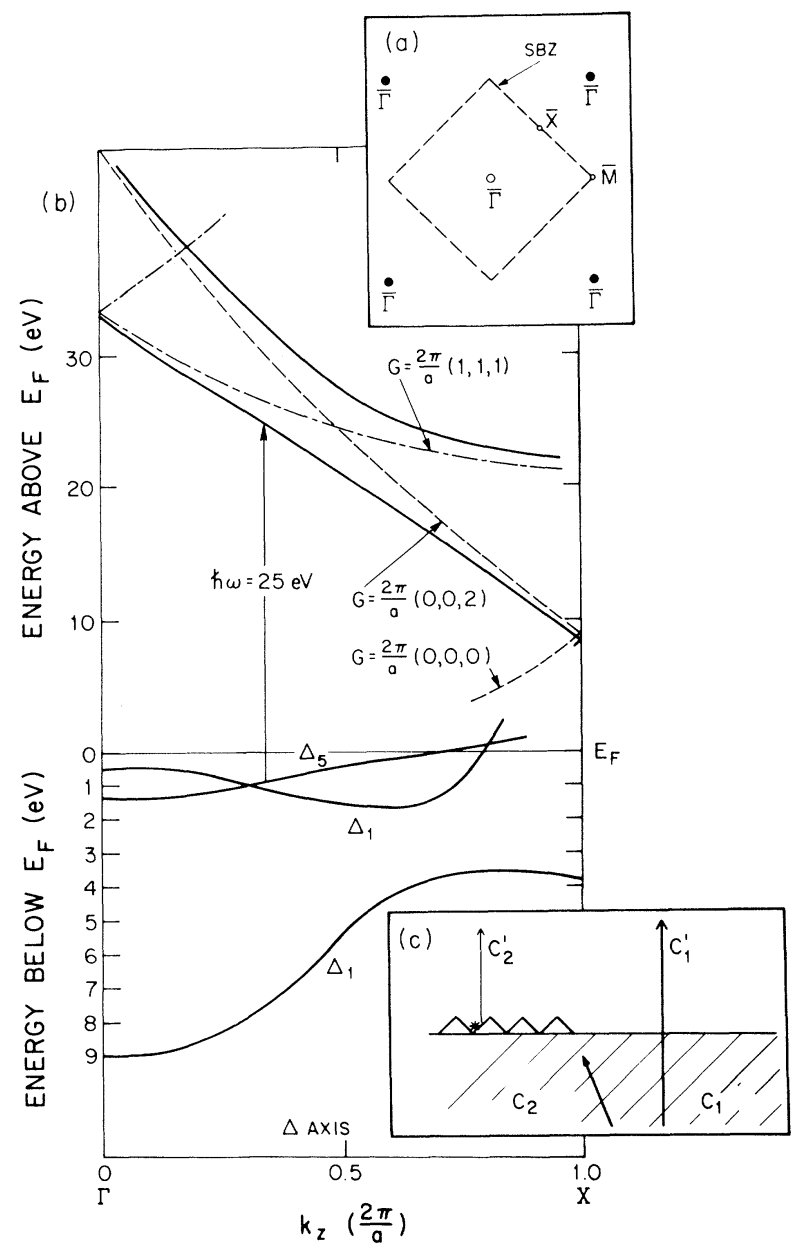

FIG. 3. (a) Picture of the LEED pattern and the surface Brillouin zone of $\mathrm{Ni}(100)$, (b) band structure of $\mathrm{Ni}$ in the $\Gamma-X$ direction, (c) schematic picture of the two channels for photoexcitation at $\hbar \omega=25 \mathrm{eV}$.

fold degenerate $\Delta_{5}$ bands by the crystal field. The $\Delta_{1}$ band from the $G=(2 \pi / a)(1,1,1)$ band is degenerate with the $\Delta_{1}$ band from the $G=(2 \pi /$ a) $(0,0,2)$ reciprocal lattice vector in the middle of the zone. Since they have the same symmetry the two bands will hybridize. This is shown by the solid lines in the top of Fig. 3(b), which were calculated with the magnitude of the Fourier coefficient of the crystal potential as a parameter. The splitting was adjusted until the bands looked like those calculated by Szmulowicz and Pease using an augmented plane-wave program. ${ }^{6}$

The hybridized bands (solid lines) are composed of only two plane waves:

$$
\begin{aligned}
\psi_{f}(k)=C_{1}(k) \exp \left\{i\left[\overrightarrow{\mathrm{e}}_{z} k_{z}-G(0,0,2)\right] \cdot \overrightarrow{\mathrm{r}}\right\} \\
+C_{2}(k) \exp \left\{i\left[\overrightarrow{\mathrm{e}}^{z} k_{z}-G(1,1,1)\right] \cdot \overrightarrow{\mathrm{r}}\right\}
\end{aligned}
$$

with the coefficients $C_{1}$ and $C_{2}$ varying as $k$ moves across the reduced zone. For example the lower of the two bands is almost all $C_{1}$ at the zone boundary $(X)$ and gradually changes character to be nearly all $C_{2}$ at $\Gamma$. Now consider the possible transitions for an exciting photon energy of $25 \mathrm{eV}$. The vertical line shows that a transition of either the $\Delta_{1}$ or $\Delta_{5}$ initial band is possible for an initial $k=0.28(2 \pi / a)$. At this point in the Brillouin zone the final band has $C_{1}=0.4$ and $C_{2}=0.9$. The component of the final band characterized by $C_{1}$ is a wave propagating in the $z$ direction, i.e., normal to the surface [see Fig. 3(c)]. The component of the final state characterized by $C_{2}$ travels in a direction not parallel to the $z$ axis, with a component of $k$ parallel to the surface equal to $(2 \pi / a)(1,1,0)$. This is a wave that would normally be observed at $\bar{\Gamma}$ in the second Brillouin zone of the surface [Fig. 3(a)].

The excited electron inside the solid has a kinetic energy with respect to the Fermi energy of $25 \mathrm{eV}$ minus the binding energy of the initial state, i.e., $\sim 24 \mathrm{eV}$. This energy is close to the energy required for the wave emitted at $\bar{\Gamma}$ in the second Brillouin zone to propagate parallel to the surface. Since $k_{\|}$is conserved in crossing the surface, the energy for this $C_{2}$ wave to be parallel to the surface is given by

$$
E_{\|}=\left(\hbar^{2} / 2 m\right)\left(k_{\|}\right)^{2}=24.2 \mathrm{eV} .
$$

Since the work function is approximately $5 \mathrm{eV}$ the wave $C_{2}$ will not be emitted from the crystal. Normally such a wave would be reflected from the surface, but in this energy region McRae et al. ${ }^{7}$ have shown that there is a surface resonance. The electron is trapped in the image potential at the surface in a state very similar to a Rydberg state. ${ }^{7}$ It is traveling along the surface with a small component of momentum perpendicular to the surface and with $k_{\|}=(2 \pi / a)(1,1,0)$ or any equivalent $k$. Schematically we have illustrated this wave traveling down the surface in Fig. 3(c). The surface resonance may be destroyed by scattering from surface imperfections or by an umklapp process. If the electron scatters from an impurity presumably the scattering is more or less isotropic. In contrast, if the scattering involves a surface umklapp process then the wave can come out normal to the surface, since its value of $k_{\|}$is equal to a surface reciprocal lattice vector. We believe that it is this effect that makes the observed spectral features so surface sensitive and explains why they have not been reported previously.

This process which involves a surface resonance and umklapp scattering (surface reciprocal lattice 
vector) creates two channels for exciting an initial state characterized by $E\left(k_{i}\right)$ into a final state characterized by a plane wave traveling normal to the surface. The amplitudes of the waves in the two channels should be summed and the intensity calculated by squaring. Therefore we can have either constructive or destructive interference between the two waves. Figure 1 indicates that for $\hbar \omega=25 \mathrm{eV}$ the interference is primarily destructive. As the photon energy is increased or decreased the wave incident onto the surface is not trapped in the surface resonance and the interference goes away.

We have observed that hydrogen, as distinct from $\mathrm{Br}, \mathrm{N}_{2}$, or $\mathrm{CO}$, does not destroy the surface resonance. No alteration of the clean $\mathrm{Ni}(100)$ interference structure was observed at $25-\mathrm{eV}$ photon energy upon hydrogen adsorption. This may be a consequence of the fact that hydrogen is a weak scatterer of electrons or of the fact that the hydrogen atoms sit relatively deeply within the fourfold site of the $\mathrm{Ni}(100)$ surface. ${ }^{9}$

There have been several observations using other techniques of surface states or resonances on $\mathrm{Ni}(100)$ above the Fermi energy. McRae et al. ${ }^{7}$ reported a scattering resonance using LEED with a binding energy of $3.5 \mathrm{eV}$ below the vacuum level for $k_{\|}=(2 \pi / a)(1,1,0)$ [i.e., $k_{\|}=0$ ]. Johnson and Smith ${ }^{10}$ have observed a state in inverse photoemission at a binding energy of $4.0 \mathrm{eV}$ below the vacuum level at $k_{\|}=0$. They suggest that the peak at $4.0-\mathrm{eV}$ binding energy is due to a bulk transition but it also agrees well with the surface resonance observed by McRae et al. ${ }^{7}$ They point out that experiments need to be done with variable photon energy detection to really separate the surface and bulk states. If we assume that the surface wave that we see is the same as the one seen by McRae et al. ${ }^{7}$ and that Johnson and Smith's peak ${ }^{10}$ is also the same state then the energies of our experiment coupled with their binding energy in the normal direction gives a value of the parallel energy inside of the crystal of $22.6 \mathrm{eV}$. This should be compared to the value of $24.2 \mathrm{eV}$ for a free electron with this value of $k$. It is not unreasonable to expect a 1.6$\mathrm{eV}$ shift in the energy due to the crystal potential. We clearly need better theoretical calculations for surface states trapped in the image potential, since this state is not a surface state by a surface resonance. ${ }^{10}$ It would also be very enlightening to have high-resolution, variable-photon-energy, inverse photoemission data on this system. Our experiments indicate that the lifetime of the surface wave decreases significantly with small amounts of contamination.

We would like to thank the staff of the Synchrotron Radiation Center of the University of Wisconsin, Madison, for their excellent support. The Tantalus I storage ring is supported by National Science Foundation Grant No. DMR 77-21888. This research was supported by the Deutsche Forschungsgemeinschaft/Sonderforschungsbereich 6 , the University of Cornell Material Science Center under National Science Foundation Grants No. NSF-DMR 82-17227 and No. NSF-DMR 83-12742, National Science Foundation Grant No. DMR 8109261, and by the Office of Naval Research. We would like to thank John Wilkins for many useful discussions.

(a) Present address: Department of Physics, Syracuse University, Syracuse N.Y. 13210.

(b) Present address: Department of Chemistry, Kyoto University, Kyoto, Japan.

${ }^{1}$ W. Eberhardt and E. W. Plummer, Phys. Rev. B 21, 3245 (1980).

${ }^{2}$ F. J. Himpsel, Appl. Opt. 19, 3964 (1980).

3J. L. Erskine, Phys. Rev. Lett. 45, 1446 (1980).

${ }^{4}$ E. W. Plummer and W. Eberhardt, Phys. Rev. B 20, 1440 (1979).

${ }^{5}$ F. J. Himpsel, J. A. Knapp, and D. E. Eastman, Phys. Rev. B 19, 2919 (1979).

${ }^{6}$ F. Szmulowicz and D. M. Pease, Phys. Rev. B 17, 3341 (1978).

${ }^{7}$ E. G. McRae, D. Aberdam, R. Baudoing, and Y. Gauthier, Surf. Sci. 78, 518 (1978).

${ }^{8} \mathrm{M}$. Grunze, P. A. Dowben, and R. Jones, Surf. Sci. 141, 455 (1984).

${ }^{9}$ S. Anderson, Chem. Phys. Lett. 55, 185 (1978).

10P. D. Johnson and N. V. Smith, Phys. Rev. B 27, 2527 (1983); D. F. Woodruff, N. V. Smith, P. D. Johnson, and W. A. Royer, Phys. Rev. B 26, 2943 (1982). 